\title{
CAPILLARY GAS CHROMATOGRAPHIC SEPARATION OF CARBOXYLIC ACIDS USING AN ACIDIC WATER STATIONARY PHASE
}

\author{
by \\ Ernest Darko and Kevin B. Thurbide* \\ Department of Chemistry, \\ University of Calgary, \\ 2500 University Drive, NW, \\ Calgary, Alberta, Canada. \\ T2N 1N4
}

Submitted for publication as an Original Article in:

Chromatographia

*Corresponding Author

Phone: (403) 220-5370

Fax: (403) 289-9488

E-mail: thurbide@ucalgary.ca 


\begin{abstract}
An acidic water stationary phase is used for the analysis of carboxylic acids in capillary gas chromatography (GC). Under regular pH 7 operating conditions, these analytes are largely ionized and elute poorly, if at all, from the water phase. However, by adjusting the phase near to $\mathrm{pH}$ 2, it is found that various acids are neutralized and can be readily eluted and separated in the system. Sulfamic acid is found to provide a stable $\mathrm{pH}$ for the water phase over time, whereas hydrochloric acid and other more volatile additives quickly evaporate from the column. Under optimal low pH conditions, the acidic analytes yield good peak shape and are readily observed for masses investigated down to 5 ng on-column. By comparison, on a conventional non-polar capillary GC column, the same analytes display 3-fold more peak tailing and are not detected for masses below about 30 ng on-column. Through altering the phase $\mathrm{pH}$, it is found that the selectivity between certain analytes can be potentially enhanced depending on their respective pKa values and/or ionizability. The analysis of various different samples containing carboxylic acids is demonstrated and the results indicate that this approach can possibly offer unique and beneficial selectivity in such determinations.
\end{abstract}

Keywords: gas chromatography; acidic; water; stationary phase; carboxylic acids 


\section{INTRODUCTION}

Gas chromatography (GC) is a very widely used separation technique that provides an effective tool for the analysis of volatile organic compounds [1-3]. Over the years it has been employed in a number of important areas such as industrial quality control, environmental monitoring, forensic analysis, and oil exploration [4-8]. Considering its excellent sensitivity and performance in such applications, advancements in GC are continuously being developed in order to further improve its field of use.

Stationary phase development is a very important area in GC since it can greatly improve separations. For example, conventional fused silica capillary GC columns are often coated with non-polar phases such as dimethylpolysiloxane, or polar phases such as polyethylene glycol [9, 10]. However, the creation of novel stationary phases that can provide more thermal stability and/or greater analyte selectivity has increasingly been a focus of research in this area. For instance, a range of novel GC stationary phase coatings have been explored, which possess very useful separation characteristics, such as those composed of ionic liquids [11-14], metal-organic frameworks [15, 16], and dithienyl benzothiadiazoles [17].

Recently, we introduced the use of water as a novel stationary phase in capillary GC [18]. In this method, water is coated on the inner wall of a stainless steel capillary and is used successfully for GC separations in the direct analysis of various compounds in both aqueous and organic samples. The technique demonstrates good efficiency and reliable performance over a range of conditions [18]. Further, the water stationary phase provides notable selectivity towards many polar analytes and little affinity for non-polar analytes. To this end, retention time on the stationary phase also appears to be considerably influenced by analyte water solubility, but is relatively independent of analyte boiling point. Therefore, considering its interesting properties, further exploration and development of this technique would be potentially beneficial. 
One challenging aspect of working with the water stationary phase system is that the GC analysis of ionizable analytes is quite difficult. For example, many organic acids and bases either elute with very poor peak shape or do not appear to elute at all under normal conditions. This is because they can exist in a largely ionized state within the typically neutral $\mathrm{pH} 7$ water coating used. As such they are heavily partitioned into the aqueous phase and poorly mobilized within the system. To address this, we recently reported on the use of a basic water stationary phase to assist in the analysis of organic bases by this method [19]. By adjusting the phase to around $\mathrm{pH}$ 11, it was found that direct analysis of organic bases was possible and good peak shape and selectivity was readily attained [19]. While an analogous approach could address similar concerns for analyzing organic acids by this method, this has not yet been investigated.

Certainly the analysis of organic acids is very important in areas such as the food industry [20-22], the petroleum industry [23, 24], and the environment [25-28], where conventional GC is often used to determine such compounds. However, GC analysis of organic acids is challenging since they often bind strongly to column active sites, which can impede detection limits and lead to poor peak shapes $[29,30]$. As a result, such acids are often derivatized in advance to relatively non-polar moieties to help reduce such unfavorable interactions [29, 30]. However, despite the benefits of this, it can be time-consuming, laborious, and a frequent source of error [20]. Thus, exploring new ways to analyze such acids more directly and simply in GC is of great interest.

Here we explore adjusting the $\mathrm{pH}$ of a water stationary phase as a novel method to separate carboxylic acids in capillary GC. Using an acidic water phase, many such analytes are rendered neutral and can be directly analyzed by this approach. The general operating parameters, separation characteristics, and analyte selectivity of the method are explored. Finally, it is applied to the direct analysis of various samples in order to gauge its effectiveness. 


\section{EXPERIMENTAL}

\section{Instrumentation and Operation}

The instrumental setup used here has been detailed previously [18, 19]. Briefly, an HP 5890-Series II GC instrument (Agilent, Palo Alto, CA, USA) equipped with a flame ionization detector (FID) was employed in these experiments. The carrier gas was high purity Helium (Praxair, Calgary, Canada). It was saturated with water vapour using an ISCO model 100DX syringe pump (Teledyne ISCO, Lincoln, NE, USA) that delivered water through a zero dead volume tee union (Vici-Valco, Houston, TX, USA) connected to the Helium line. A 1 m stainless steel (SS) pre-heating coil (1/16” O.D. x 250 m I.D.; Chromatographic Specialties, Brockville, ON, CAN) was led out of the tee union and used to equilibrate the water vapor and carrier gas. Both the tee union and coil were housed inside the oven. The outlet of the coil was connected to the injector. Normally, this setup directed $1-3 \mu \mathrm{L} / \mathrm{min}$ of water humidification to the column carrier gas in order to prevent stationary phase loss and provide stable analyte retention times.

The separation column was connected to the injector, and typically operated with about $50 \mathrm{~cm} / \mathrm{s}$ of carrier gas and a 10:1 split ratio. An $8 \mathrm{~m}$ length of 316 SS capillary tubing (1/16” O.D. x $100 \mu \mathrm{m}$ I.D.; Chromatographic Specialties) was normally used for a separation column, while a 2 m length of the same (at flows near $20 \mathrm{~cm} / \mathrm{s}$ ) was also used in some preliminary work. The column outlet was joined to a $50 \mathrm{~cm}$ SS capillary restrictor (1/16” O.D. x 75 $\mu \mathrm{m}$ I.D.) via a zero dead volume union (Vici-Valco). This created system backpressure, which promoted phase stability at higher temperatures. The restrictor outlet was positioned inside the FID burner just below the flame. An injector/detector temperature near $220{ }^{\circ} \mathrm{C}$ was maintained during the experiments. High purity Hydrogen and Air (Praxair) were used to support the detector flame at 
respective flows of 90 and $350 \mathrm{~mL} / \mathrm{min}$. For comparisons, a conventional DB-1 column (dimethyl polysiloxane phase; $250 \mu$ m I.D. x 30 m; Agilent) was also used.

\section{Stationary Phase Preparation}

Normally, stock solutions of the acids investigated for use in stationary phase coating were prepared in HPLC-grade water at about $0.02 \mathrm{M}$. A pH meter, after calibration with an appropriate buffer, was used to measure the $\mathrm{pH}$ of the stirred stock solution. In cases where an intermediate $\mathrm{pH}$ was required, a portion of the stock solution was stirred and a few drops of dilute base were slowly added until the desired $\mathrm{pH}$ was achieved. This $\mathrm{pH}$-adjusted solution was then used immediately to coat the SS capillary column as described previously [18]. Following this, the column was mounted inside the GC oven for use.

\section{Chemicals and Reagents}

Solvents used include butanol, heptane, acetone, ethanol ( $\geq 99 \%$ each; Sigma-Aldrich, Canada), and HPLC-grade water (Honeywell Burdick \& Jackson, Muskegon, USA), which was also used to prepare coating solutions. Analyte acids including acetic ( $\geq 97 \%$; Fisher Scientific, USA), propionic ( $\geq 99.5 \%$; Fluka, Germany), isobutylphenylpropionic ( $\geq 98.0 \%$; Fluka), benzoic, octanoic, heptanoic, hexanoic, 3-methylbutanoic, pentanoic, butanoic, cyclohexane carboxylic, and 3-pentenoic (all $\geq 97 \%$; Sigma-Aldrich) were used to prepare standards in butanol and other solvents at about $10 \mu \mathrm{g} / \mu \mathrm{L}$. A mouthwash and an aftershave sample were each spiked with benzoic acid (about $10 \mu \mathrm{g} / \mu \mathrm{L}$ ). A mixture of alkyl carboxylic acids was prepared in canola oil (10 $\mu \mathrm{g} / \mu \mathrm{L}$ each). An ibuprofen tablet (200 mg) was ground and extracted from acidified aqueous solution into heptane. A mixture of naphthenic acids (Sigma-Aldrich) was prepared in automotive fuel $(15 \mu \mathrm{g} / \mu \mathrm{L})$. All commercial samples were purchased from local vendors. All other variations are described in the text. 


\section{RESULTS AND DISCUSSION}

\section{General Operating Characteristics}

Preliminary efforts were made to establish the extent to which lowering the water stationary phase $\mathrm{pH}$ may assist in the elution of carboxylic acids. Fig. 1 demonstrates the typical results obtained when using $\mathrm{HCl}$ to decrease the phase $\mathrm{pH}$. As seen in fig. 1a, under regular conditions of a neutral water phase at $\mathrm{pH} 7$, the peak obtained for an injected hexanoic acid analyte is very broad and poorly discernable. At this $\mathrm{pH}$, about 99\% of the hexanoic acid (pKa 4.8) is in the ionized form. As such, it is heavily partitioned to the water stationary phase and difficult to elute well. In contrast to this, as the $\mathrm{pH}$ of the water phase is lowered to 4.0 (fig. 1b), about $13 \%$ of the analyte is ionized and an improved peak shape emerges. Finally, in fig. 1c the $\mathrm{pH}$ is reduced to 2.2 , where only about $0.25 \%$ of the analyte is ionized. As a result, a much sharper and well-defined peak is observed. Therefore, decreasing the $\mathrm{pH}$ of the water stationary phase can greatly facilitate the ability of the system to analyze such organic acids. In our experience, it was found that reducing the $\mathrm{pH}$ about 2 or more units below the analyte pKa was sufficient to produce reasonable peaks shapes and so this was followed in subsequent trials.

Although using $\mathrm{HCl}$ to lower the $\mathrm{pH}$ of the water stationary phase was advantageous, it became apparent in early trials that the improved peak shape readily eroded again over time. This is likely due to the volatility of $\mathrm{HCl}$ and the fact that it can escape from the column during experiments, causing the $\mathrm{pH}$ of the water stationary phase to rise again. Incidentally, it was wondered if the short $2 \mathrm{~m}$ column length initially used in those trials might have also played a possible role in this. However it was found that longer columns also displayed this same phenomenon. Fig. 2 demonstrates the erosion of a hexanoic acid peak over time when using $\mathrm{HCl}$ to adjust the $\mathrm{pH}$ of the water phase. As can be seen in fig. 2a, upon establishing the column, the 
first injection produces a prominent, well-defined peak for the analyte. However, only 20 minutes later (fig. 2b) the peak shape is badly deteriorated. In fact, after about 40 minutes of operation (fig. 2c), the peak is barely discernable.

Given these results, efforts were made to identify another acid that might be more suitable for this purpose. Initial attempts explored nitric acid and formic acid, however it was found that due to their volatility, neither was able to provide a consistently favorable peak shape over time. Next, stronger and less volatile additives such as sulfuric acid and phosphoric acid were examined. Both of these provided a stable water phase $\mathrm{pH}$ over time. However, under the typically hot column conditions employed, each was found to considerably erode the SS capillary wall after only a few trials, which in turn degraded peak shapes and did permanent damage to the surface. Finally, sulfamic acid was investigated. As a non-volatile additive it was found to provide a stable $\mathrm{pH}$ in the water stationary phase over time, even at higher carrier gas velocities, making system operation much more flexible. Further, it was also not observed to produce any adverse effects on the SS capillary wall. Fig. 3 shows an example of this for a hexanoic acid separation using sulfamic acid to adjust the water phase $\mathrm{pH}$. As seen in the figure, a reasonably sharp and well-defined peak for the analyte is still readily obtained even after 80 minutes of operation at a carrier gas velocity of about $50 \mathrm{~cm} / \mathrm{s}$. This also translated into useful reproducibility as both repeated injections and day to day experiments produced analyte retention times that varied within about 1\% RSD $(n=3)$. Therefore, given its favorable properties, sulfamic acid was used exclusively going forward.

\section{Analyte Retention}

To further probe the retention characteristics of the acidic water stationary phase, an assortment of carboxylic acids was surveyed in the system. Table 1 displays the typical results of these investigations with the retention time obtained for the various analytes under isothermal 
conditions. As can be seen, a wide range of retention is observed for the compounds investigated, which are listed in increasing elution order and include primary/secondary aliphatic, aromatic, cyclic, and saturated/unsaturated carboxylic acids.

Examination of the data reveals some interesting separation characteristics. For instance, similar to previous work with the water stationary phase $[18,19]$, the analytes appear to follow somewhat of a normal phase type of retention pattern, where more polar solutes tend to elute much later than those less polar. This is illustrated with the n-alkyl carboxylic acid series eluting in order of increasing polarity from octanoic to acetic acid. As well, it is also apparent that analyte boiling point (also included in the table) has little influence on the retention observed. For example, acetic acid is much smaller than octanoic acid and has a boiling point nearly half as large, yet it is almost 10 fold more retained. Conversely though, as also noted previously [18, 19], elution order does seem to be considerably influenced by analyte water solubility, which is found to generally increase with retention for the compounds examined [31]. For instance, water solubility rises nearly 100 times in the transition from octanoic to acetic acid [31].

Still, some exceptions to these observations can also be noted. For instance, heptanoic acid and cyclohexane carboxylic acid each have very similar molecular formulas, boiling points, and water solubilities [31]. Despite this, however, the latter is notably more retained. This is also true of pentanoic acid and 3-pentenoic acid. While the reasons for this are not entirely clear, it could likely be related to core structural differences between the molecules. For instance, in this regard it is interesting to note that certain unsaturated analytes have previously shown enhanced retention on a boric acid impregnated silica stationary phase [32]. As such, further investigations would be required to better understand this in the future. None the less, overall the data appear to indicate that more polar, water soluble acids tend to be more retained by this method and that it 
displays a reasonable ability to separate such analytes. Fig. 4 illustrates this with the separation of a mixture of carboxylic acids. As seen, prominent peaks with good shape are produced for the various acids, which are well separated in the system.

To gain some additional context of the properties observed, a comparison was made between separations performed on a conventional (unhydrated) capillary GC column and the current acidic water stationary phase system. As somewhat anticipated, it was found that the carboxylic acid analytes consistently displayed excessive tailing in their peak shape when analyzed on the conventional column. Further, no peak could be observed under any condition examined for analyte amounts below about $30 \mathrm{ng}$ on-column. This is understandable since it is well known that such acids can bind strongly to residual active sites on conventional GC columns and often require derivitization as a result [29]. Fig. 5a shows the typical result obtained for a hexanoic acid analyte on the conventional GC column. As seen, the 60 ng injection produces a very broad, asymmetrical analyte peak with a tailing factor of about 4.4. By comparison, acid analytes on the acidic water stationary phase presented much more symmetrical peak shapes and could be readily observed for injected amounts that were 10 times lower. Fig. 5b demonstrates this with the peak obtained for a $5 \mathrm{ng}$ injection of hexanoic acid on the acidic water stationary phase. As seen, a notable peak appears with good shape and a greatly reduced tailing factor near 1.3. Therefore, this approach could potentially provide beneficial sensitivity and improved peak symmetry in the analysis of such acids.

\section{Altering Selectivity}

Since the $\mathrm{pH}$ of the water stationary phase can directly control analyte ionization and potentially alter retention characteristics, this presents the possibility of using this parameter to change analyte selectivity on the column. For instance, if two analytes have sufficiently different 
pKa values, or if one is non-ionizable, then their relative retention could be impacted by changing the water phase pH. Fig. 6 demonstrates this with the separation of ethanol and octanoic acid. As seen in fig. 6A, when the stationary phase is at pH 2.2, octanoic acid (pKa 4.9) is almost completely neutral and readily elutes very close to the ethanol peak with a retention time of about 4 minutes. Conversely, when the phase is set to a pH of 7 (fig. 6B), octanoic acid is near fully ionized. As such it is heavily retained on the water stationary phase and disappears from the retention window, leaving only the neutral ethanol peak. Thus, for certain analyte pairs, chromatographic selectivity in this method could be potentially tunable if appropriate adjustments are made to the water stationary phase $\mathrm{pH}$. Accordingly, such an advent could be beneficial, for instance, in facilitating analyte resolution within complex matrices.

\section{Applications}

A number of different samples were investigated using the acidic water stationary phase system in order to probe its ability to analyze for carboxylic acids within various types of matrices. Fig. 7 shows some examples of these. The chromatogram from a neat injection of commercial white vinegar is displayed in fig. 7A. As seen, a strong, well-defined peak for acetic acid is observed near the 30 minute mark against a reasonably smooth background. Additionally a small contribution from residual ethanol is also observed at around 3 minutes. Conventional GC analysis of vinegar frequently requires lengthy derivitization steps and extraction into an organic solvent for determining the acids present [33]. However, since the acidic water stationary phase is readily compatible with aqueous samples, the vinegar can be injected directly here. Similar results were also obtained when directly analyzing small chain carboxylic acids in a canola oil matrix. Therefore this can be potentially useful for reducing sample preparation steps. 
Fig. 7B displays the chromatogram from a neat injection of aftershave cologne. Benzoic acid is known for its anti-bacterial and anti-fungal properties even at low concentrations and so it is often used as a preservative in food and cosmetics [34]. As shown in the figure, after the elution of ethanol (the primary solvent of the formulation) and a number of other components, the benzoic acid peak clearly appears at around 40 minutes. Again, this is useful since conventional GC analysis often requires laborious derivatization of benzoic acid first [34].

Ibuprofen (i.e. isobutylphenylpropionic acid) is a common anti-inflammatory drug that is widely available in a variety of capsule or tablet formulations, and it is commonly analyzed by conventional GC after extensive derivitization procedures [35]. Fig. 7C shows the chromatogram for a heptane extract of an acidified aqueous solution containing a ground ibuprofen tablet. As seen, the ibuprofen analyte appears as a prominent well-defined peak at around 20 minutes. It should also be noted that similar results were observed when injecting ibuprofen in either the free acid or the sodium salt form, since the water stationary phase $\mathrm{pH}$ controls the analyte form on-column. Therefore, because such drug molecules are often available in a variety of these forms and are frequently found in matrices such as waste water [35], the ability of the system to readily analyze either form of the analyte and in both organic or aqueous media can potentially facilitate such efforts.

Finally, a more complicated sample matrix was examined with the system. Naphthenic acids are a complex fraction of carboxylic acid moieties that naturally exist in oil sands deposits and are carried through to petroleum products and aqueous settling ponds after processing [36]. Since their presence can cause toxicity concerns for wildlife and corrosion issues for pipelines, analysis of these acids is an important routine procedure [36]. While conventional GC is often used for this, derivitization of the acids is normally required prior to injection [36]. Further, since 
both the acid fraction and matrix are often very complex, this complicates determinations and the analytes are usually quantified as an unresolved cluster amongst a myriad of other hydrocarbons in the petroleum extract [36]. As such, methods to simplify such analyses are of great interest.

Fig. 8A demonstrates this issue with the analysis of a commercial automotive fuel containing naphthenic acids using a conventional GC column. As shown, a multitude of peaks representing the numerous hydrocarbons in the fuel continually elute over the 20 minute separation. Examination of the naphthenic acid standard mixture used reveals that its components steadily elute from the 12 minute mark until the end of the run. Thus, due to severe overlap with other hydrocarbons, it is very difficult to discern the target acids from the matrix.

By comparison, since the main fuel sample components are highly non-polar, they are unretained by the water stationary phase, as has also been noted previously [18, 19]. Fig. 8B shows an example of this, with the chromatogram of the same automotive fuel without the naphthenic acids present as analyzed on the acidic water stationary phase. As shown, the chromatogram has a single, large off-scale peak that elutes in the void volume and represents the entire bulk of the fuel matrix. Consequently, when the naphthenic acids are present (fig. 8C), they are retained well beyond this and elute as a complex mixture containing some reasonably sharp and well-defined peaks. Therefore, the ability of the acidic water stationary phase system to analyze such samples directly and with enhanced selectivity over the bulk hydrocarbon matrix could be useful in these and other related determinations.

\section{CONCLUSIONS}

An acidic water stationary phase has been demonstrated and characterized for the direct analysis of carboxylic acids. Using a low $\mathrm{pH}$ phase based on a non-volatile sulfamic acid 
additive, a stable separation system is provided. This approach is found to yield good peak shapes when compared to conventional GC columns and for smaller analyte masses. Through altering the phase $\mathrm{pH}$, certain improvements in separation selectivity may also be possible. Conventional practices of analyte derivitization were not required and direct injection of either aqueous or organic solvents and free or sodium salt forms of the acid could be readily accommodated. The analysis of a number of different samples demonstrates that this method can potentially facilitate such organic acid determinations by eliminating the need for a number of sample preparation steps.

\section{ACKNOWLEDGEMENT}

The authors are grateful to the Natural Sciences and Engineering Research Council of Canada (NSERC) for a Discovery Grant in support of this project.

\section{Compliance with Ethical Standards:}

Funding: This study was funded by an NSERC Discovery Grant.

Conflict of Interest: The authors declare no conflict of interest.

Ethical approval: This study does not involve any human or animal participants. 


\section{REFERENCES}

[1] Cazes J (2005) Ewing’s Analytical instrumentation handbook. Marcel Dekker, New York

[2] Venn RF (2008) In: Venn RF (ed) Principles and practice of bioanalysis, 2nd edn. CRC Press - Taylor \& Francis Group, Florida

[3] Santos FJ, Galceran MT (2003) Modern developments in gas chromatography-mass spectrometry-based environmental analysis. J Chromatogr A 1000:125-151

[4] James AT, Martin AJP (1952) Gas-liquid partition chromatography: The separation and micro-extraction of volatile fatty acids from formic acid to dodecanoic acid. J Biochem 50:679690

[5] Liska I, Slobodnik J (1996) Comparison of gas and liquid chromatography for analyzing polar pesticides in water samples. J Chromatogr A 733:235-258

[6] Kidwell DA, Kidwell JD, Shinohara F, Harper C, Roarty K, Bernadt K, McCaulley RA, Smith FP (2003) Comparison of daily urine, sweat, and skin swabs among cocaine users. Forensic Sci Int 133:63-78

[7] Conway JM, Birnbaum AK, Marino SE, Cloyd JC, Remmel RP (2012) A sensitive capillary GC-MS method for analysis of topiramate from plasma obtained from single-dose studies. Biomed J Chromatogr A 26:1071-1076

[8] Scott SA, Douglas GS, Uhler AD, McCarthy KJ, Emsbo-Mattingly SD (2005) Identifying the source of mystery waterborne oil spills - A case for quantitative chemical fingerprinting. J Environ Claim 17:71-88

[9] Li QL, Poole CF (2001) Selectivity equivalence of poly (dimethyldiphenylsiloxane) stationary phases for open-tubular column gas chromatography. J Sep Sci 24:129-135

[10] Poole CF, Li QL, Kiridena W, Koziol WW (2001) Selectivity assessment of popular stationary phases for open-tubular column gas chromatography. J Chromatogr A 912:107-117

[11] Alvarez JG, Gomis DB, Abrodo PA, Llorente DD, Busto E, Lombardia NR, Fernandez VG, Alvarez MDG (2011) Evaluation of new ionic liquids as high stability selective stationary phases in gas chromatography. Anal Bioanal Chem 400:1209-1216

[12] Shashkov MV, Sidel'nikov VN (2015) Thermostable columns based on ionic liquids for the analysis of hydrocarbon mixtures by two-dimensional chromatography. Prot Met Phys Chem+ 51:1080-1086

[13] Mendoza LG, Gonzalez-Alvarez J, Gonzalo CF, Arias-Abrodo P, Altava B, Luis SV, Burguete MI, Gutierrez-Alvarez MD (2015) Gas chromatographic analysis of fatty acid methyl 
esters of milk fat by an ionic liquid derived from L-phenylalanine as the stationary phase. Talanta 143:212-218

[14] Kulsing C, Nolvachai Y, Hugel HM, Marriott PJ (2015) Developments in gas chromatography using ionic liquid stationary phases. LC GC Eur 28:434-440

[15] Srivastava M, Roy PK, Ramanan A (2016) Hydrolytically stable ZIF-8@PDMS core-shell microspheres for gas-solid chromatographic separation. Rsc Adv 6:13426-13432

[16] Yusuf K, Aqel A, Othman ZAL (2014) Metal-organic frameworks in chromatography. J Chromatogr A 1348:1-16

[17] Sun T, Tian L, Li JM, Qi ML, Fu RN, Huang XB (2013) Dithienyl benzothiadiazole derivatives: A new type of stationary phases for capillary gas chromatography. J Chromatogr A 1321:109-118

[18] Gallant JA, Thurbide KB (2014) Properties of water as a novel stationary phase in capillary gas chromatography. J Chromatogr A 1359:247-254

[19] Darko E, Thurbide KB (2016) Capillary gas chromatographic separation of organic bases using a pH-adjusted basic water stationary phase. J Chromatogr A 1465:184-189

[20] Yang MH, Choong YM (2001) A rapid gas chromatographic method for direct determination of short-chain $\left(\mathrm{C}_{2}-\mathrm{C}_{12}\right)$ volatile organic acids in foods. Food Chem 75:101-108

[21] Jurado-Sanchez B, Ballesteros E, Gallego M (2011) Gas chromatographic determination of 29 organic acids in foodstuffs after continuous solid-phase extraction. Talanta 84:924-930

[22] Mato I, Suarez-Luque S, Huidobro JF (2005) A review of the analytical methods to determine organic acids in grape juices and wines. Food Res Int 38:1175-1188

[23] Zuo-gang G, Shu-rong W, Ying-ying Z, Zhong-yang L, Ke-fa C (2009) Separation of acid compounds for refining biomass pyrolysis oil. J Fuel Chem Technol 37:49-52

[24] Gutierrez-Villagomez JM, Vazquez-Martinez J, Ramirez-Chavez E, Molina-Torres J, Trudeau VL (2017) Analysis of naphthenic acid mixtures as pentafluorobenzyl derivatives by gas chromatography-electron impact mass spectrometry. Talanta 162:440-452

[25] Jurado-Sanchez B, Ballesteros E, Gallego M (2010) Determination of carboxylic acids in water by gas chromatography using several detectors after flow preconcentration. J Chromatogr A 1217:7440-7447

[26] Jurado-Sanchez B, Ballesteros E, Gallego M (2012) Determination of carboxylic acids in water by gas chromatography-mass spectrometry after continuous extraction and derivatization. Talanta 93:224-232 
[27] Kawamura K, Ng LL, Kaplan IR (1985) Determination of organic-acids (C1-C10) in the atmosphere, motor exhausts, and engine oils. Environ Sci Technol 19:1082-1086

[28] Khasanov VV, Makarycheva AI, Slizhov YG (2016) Determination of aliphatic acids in natural waters using distillation and solid-phase extraction. J Anal Chem 71:1028-1032

[29] Hallmann C, van Aarssen BGK, Grice K (2008) Relative efficiency of free fatty acid butyl esterification choice of catalyst and derivatisation procedure. J Chromatogr A 1198:14-20

[30] Ford QL, Burns JM, Ferry JL (2007) Aqueous in situ derivatization of carboxylic acids by an ionic carbodiimide and 2,2,2-trifluoroethylamine for electron-capture detection. J Chromatogr A 1145:241-245

[31] Yalkowsky SH, He Y (2003) Handbook of aqueous solubility data. CRC Press, Florida

[32] Fuchs B, Suss R, Teuber K, Eibisch M, Schiller J (2011) Lipid analysis by thin-layer chromatography-A review of the current state. J Chromatogr A 1218:2754-2774

[33] Pinu FR, de Carvalho-Silva S, Uetanabaro APT, Villas-Boas SG (2016) Vinegar metabolomics: An explorative study of commercial balsamic vinegars using gas chromatography-mass spectrometry. Metabolites 6:1-15

[34] Brittain HG (1999) In: Brittain HG (ed) Analytical profiles of drug substances and excipients, 1st edn. Academic Press, California

[35] Sebok A, Vasanits-Zsigrai A, Palko G, Zaray G, Molnar-Perl I (2008) Identification and quantification of ibuprofen, naproxen, ketoprofen and diclofenac present in waste-waters, as their trimethylsilyl derivatives, by gas chromatography mass spectrometry. Talanta 76:642-650

[36] Ortiz X, Jobst KJ, Reiner EJ, Backus SM, Peru KM, McMartin DW, O'Sullivan G, Taguchi VY, Headley JV (2014) Characterization of Naphthenic Acids by Gas Chromatography-Fourier Transform Ion Cyclotron Resonance Mass Spectrometry. Anal Chem 86:7666-7673 
Table 1: Retention time of various analytes on the acidic ${ }^{\mathrm{a}}$ water stationary phase

\begin{tabular}{|c|c|c|}
\hline Compound & $\begin{array}{l}\text { Retention } \\
\text { (min) }\end{array}$ & $\begin{array}{l}\text { Boiling point } \\
\left({ }^{\circ} \mathrm{C}\right)\end{array}$ \\
\hline Octanoic acid & 3.8 & 237 \\
\hline Heptanoic acid & 4.9 & 223 \\
\hline Hexanoic acid & 6.6 & 205 \\
\hline 3-Methylbutanoic acid & 7.4 & 175 \\
\hline Pentanoic acid & 8.9 & 185 \\
\hline Butanoic acid & 11.9 & 164 \\
\hline Cyclohexane carboxylic acid & 14.4 & 232 \\
\hline Propionic acid & 17.0 & 141 \\
\hline 3-Pentenoic acid & 18.4 & 192 \\
\hline Isobutylphenylpropionic acid & 20.5 & 157 \\
\hline Acetic acid & 29.9 & 118 \\
\hline Benzoic acid & 40.3 & 249 \\
\hline
\end{tabular}

a. $\mathrm{pH}=2.2 ; 250 \mu \mathrm{m}$ I.D. $\mathrm{x} 8 \mathrm{~m}$ column at $110{ }^{\circ} \mathrm{C}$; void time is $0.2 \mathrm{~min}$. 


\section{Figure Captions}

Fig. 1 Chromatograms for hexanoic acid on a water stationary phase at $\mathrm{pH}$ a) 7.0, b) 4.0, and c) 2.2. Hydrochloric acid is used to adjust the $\mathrm{pH}$. Column temperature is 110 ${ }^{\circ} \mathrm{C}$. Column length is $2 \mathrm{~m}$. Carrier gas velocity is $17 \mathrm{~cm} / \mathrm{s}$

Fig. 2 Chromatograms for hexanoic acid on a water stationary phase adjusted to $\mathrm{pH} 2.2$ with hydrochloric acid a) immediately, b) 20 minutes, and c) 40 minutes after the column was installed. Column length is $8 \mathrm{~m}$. Carrier gas velocity is $20 \mathrm{~cm} / \mathrm{s}$. Column temperature is $110{ }^{\circ} \mathrm{C}$

Fig. 3 Chromatogram for hexanoic acid on a water stationary phase adjusted to $\mathrm{pH} 2.2$ with sulfamic acid. Injection is after 80 minutes of column operation. Column length is $8 \mathrm{~m}$. Carrier gas velocity is $53 \mathrm{~cm} / \mathrm{s}$. Column temperature is $110{ }^{\circ} \mathrm{C}$

Fig. 4 Typical separation of about $1 \mu \mathrm{g}$ each of various analytes on the acidic water stationary phase ( $\mathrm{pH}$ 2.2). Column temperature is $110^{\circ} \mathrm{C}$. Elution order is butanol solvent then 1) heptanoic acid, 2) 3-methylbutanoic acid, 3) butanoic acid, 4) cyclohexane carboxylic acid, and 5) 3-pentenoic acid

Fig. 5 Chromatograms of hexanoic acid on a) a conventional DB-1 column, and b) a pH 2.2 water stationary phase column. Conditions: a) 60 ng analyte, $250 \mu \mathrm{m}$ I.D. x 30 m column at $110^{\circ} \mathrm{C}$; b) $5 \mathrm{ng}$ analyte, $250 \mu \mathrm{m}$ I.D. $\mathrm{x} 8 \mathrm{~m}$ column at $110{ }^{\circ} \mathrm{C}$

Fig. 6 Separation of a mixture of ethanol (1) and octanoic acid (2) in heptane on a water stationary phase at a) $\mathrm{pH} 2.2$ and b) $\mathrm{pH}$ 7. Column length is $8 \mathrm{~m}$. Column temperature is $110^{\circ} \mathrm{C}$

Fig. 7 Chromatograms of a) a neat injection of vinegar (5\%), b) a neat injection of aftershave containing benzoic acid (10 $\mu \mathrm{g})$, and c) a heptane extract of a ground ibuprofen tablet $(200 \mathrm{mg})$. Column temperature is $110^{\circ} \mathrm{C}$. Column length is $8 \mathrm{~m}$

Fig. 8 Chromatograms for neat injections of an automotive fuel sample with (a, c) and without (b) naphthenic acids (15 $\mu$ g) present. (a) is analyzed on a conventional DB-1 column ( $250 \mu \mathrm{m}$ I.D. x $30 \mathrm{~m}$; 25 to $180{ }^{\circ} \mathrm{C}$ at $5{ }^{\circ} \mathrm{C} / \mathrm{min}$ ). (b and c) are analyzed on a pH 2.2 water stationary phase $\left(8 \mathrm{~m}\right.$ column at $\left.90^{\circ} \mathrm{C}\right)$ 

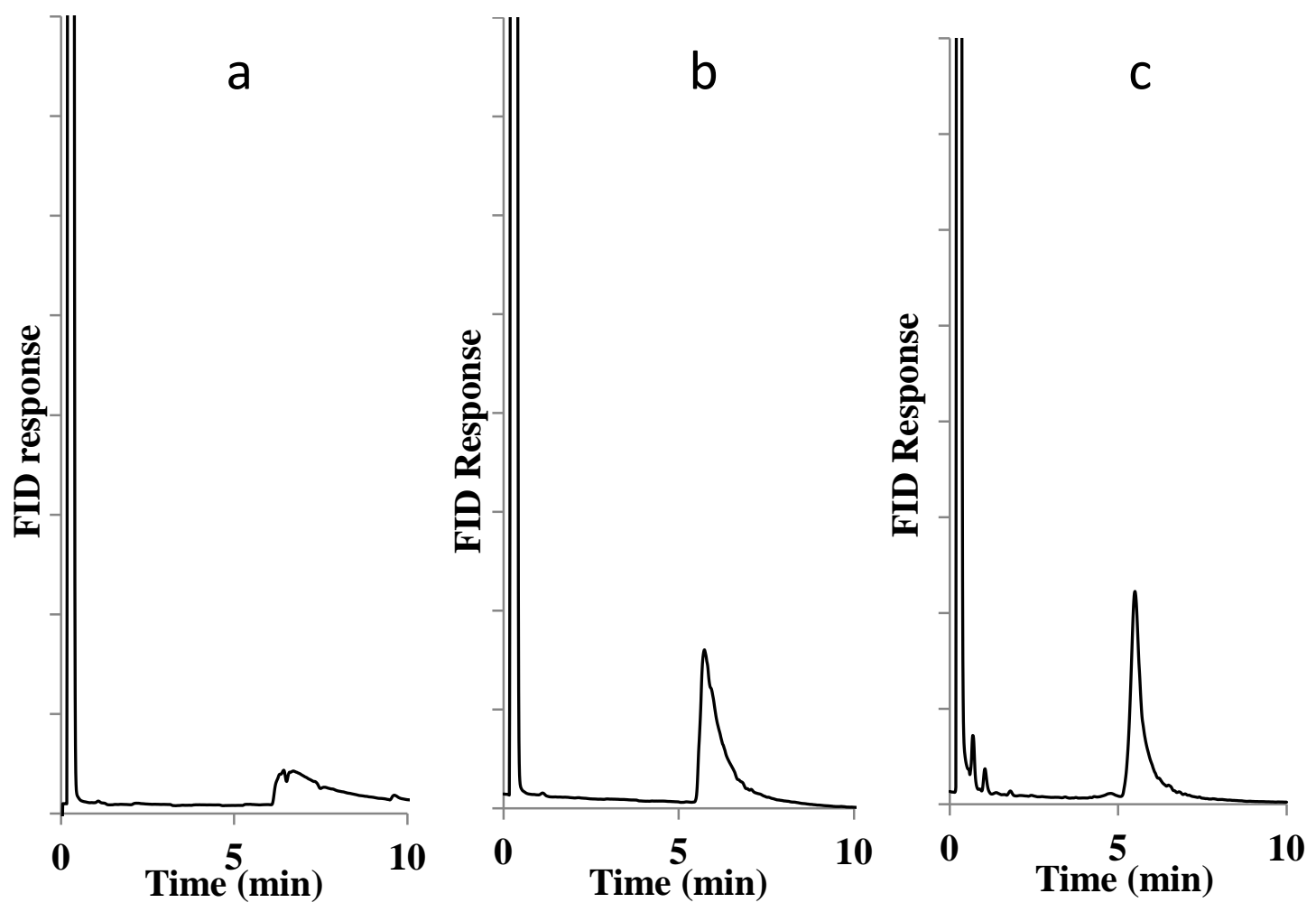

Fig. 1 

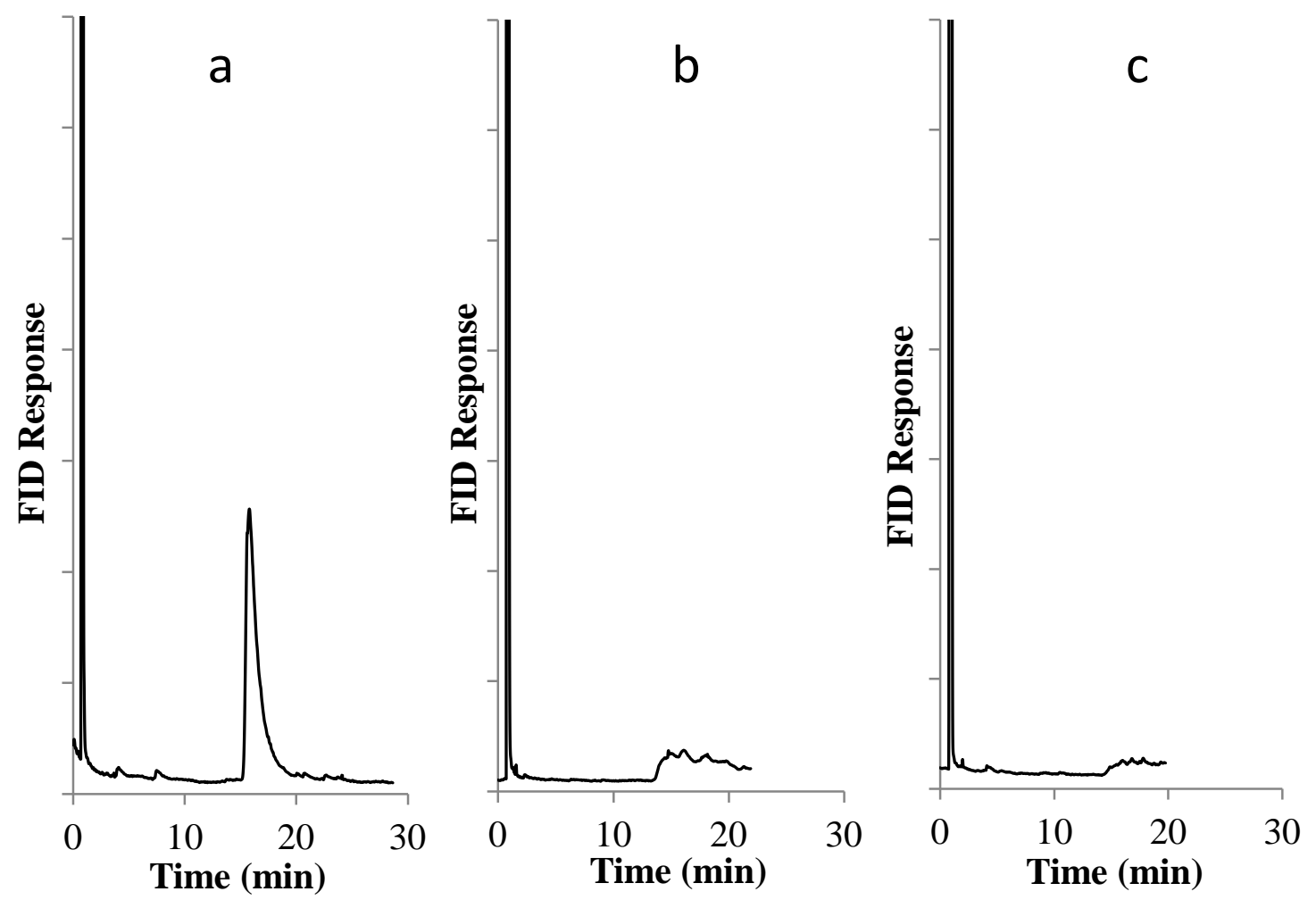

Fig. 2 


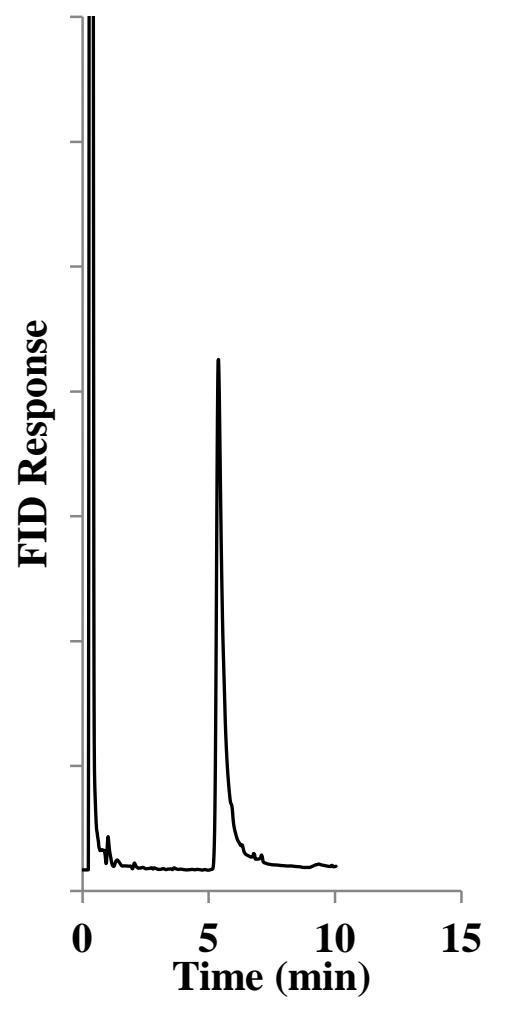

Fig. 3 


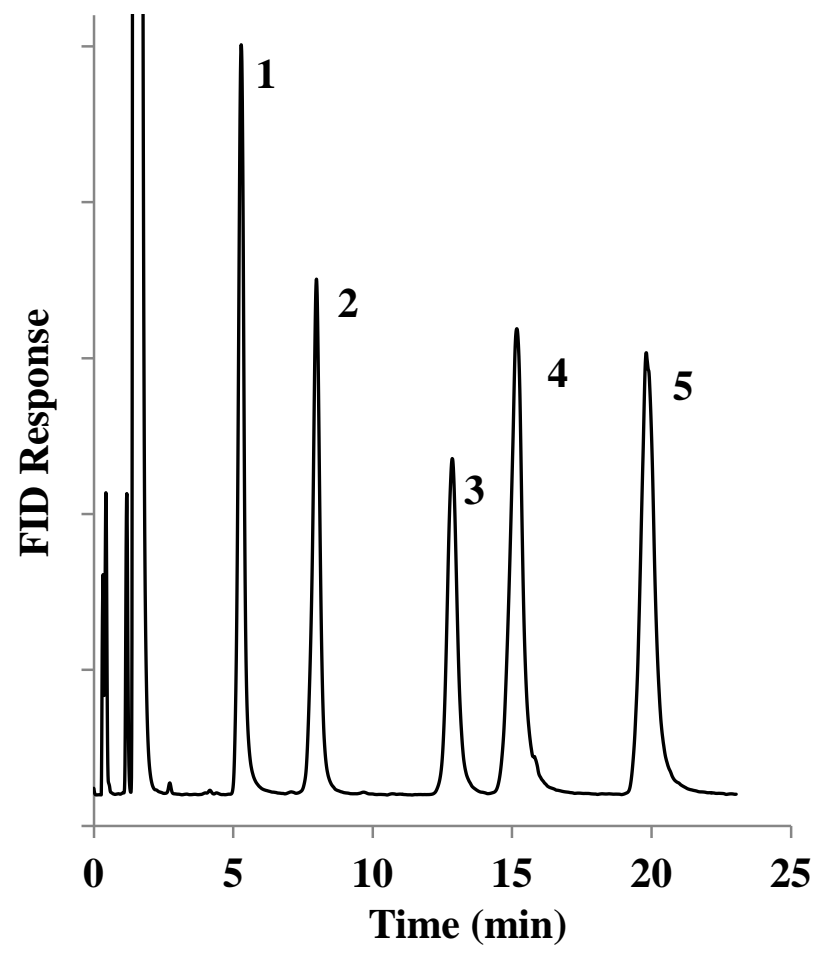

Fig. 4 

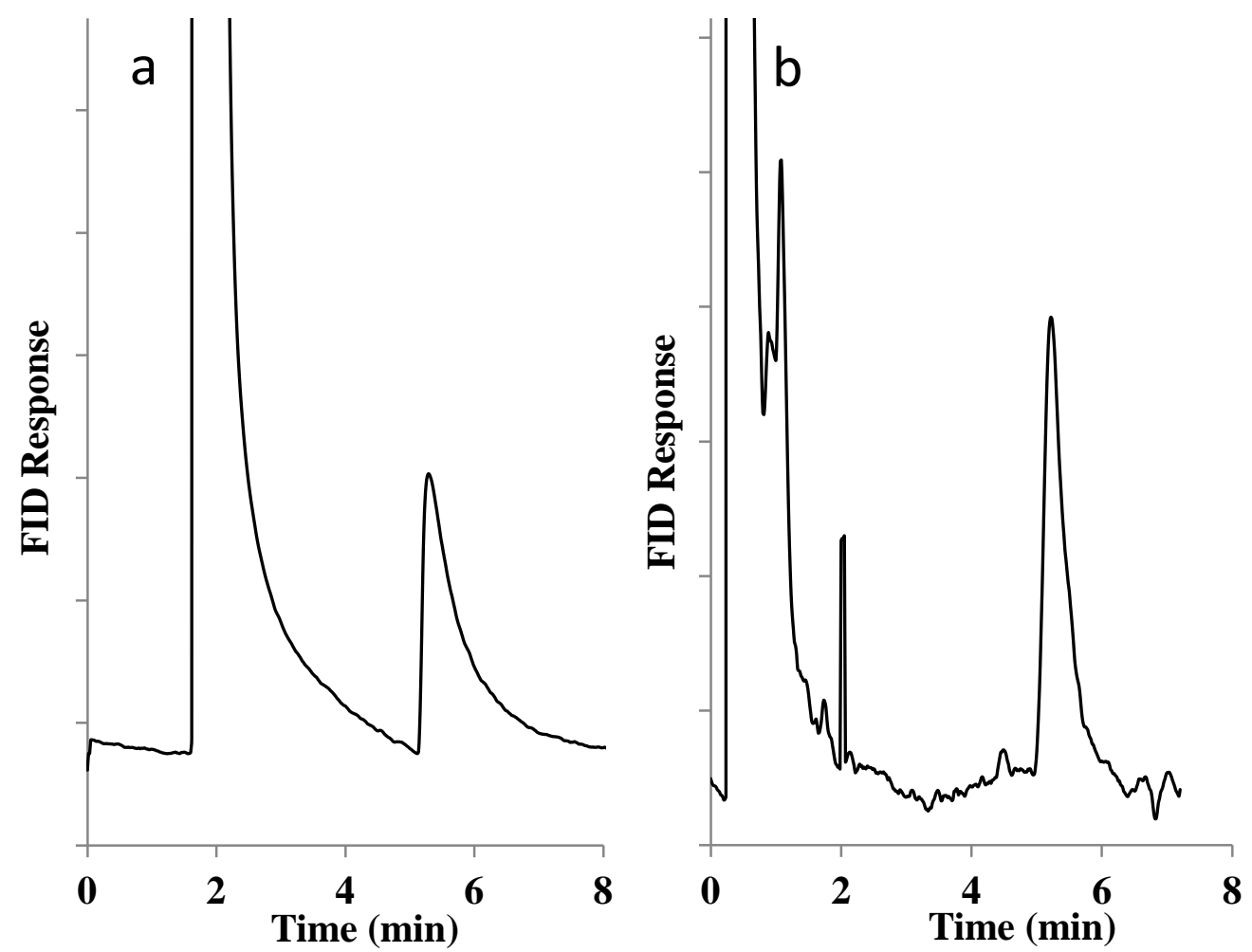

Fig. 5 

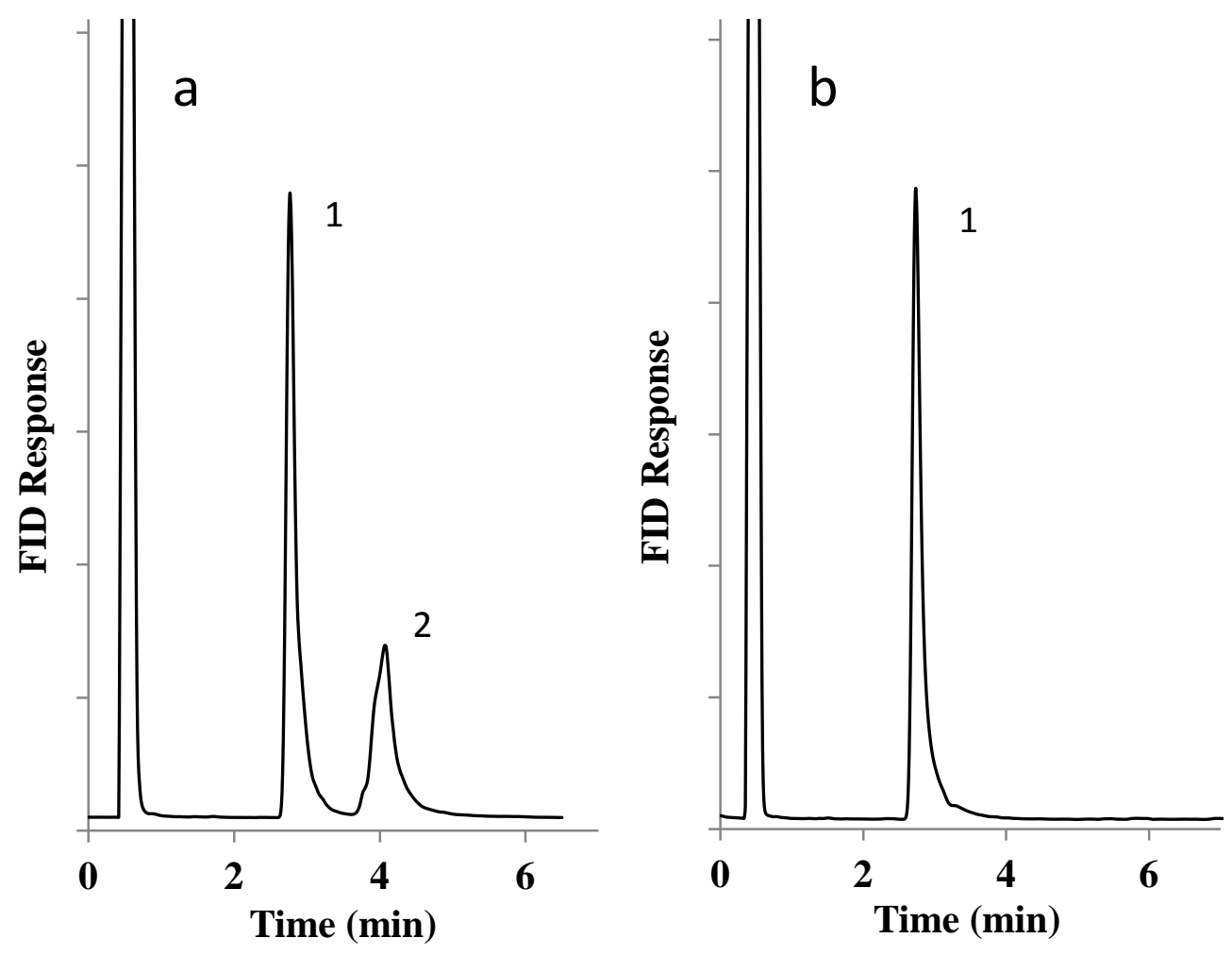

Fig. 6 


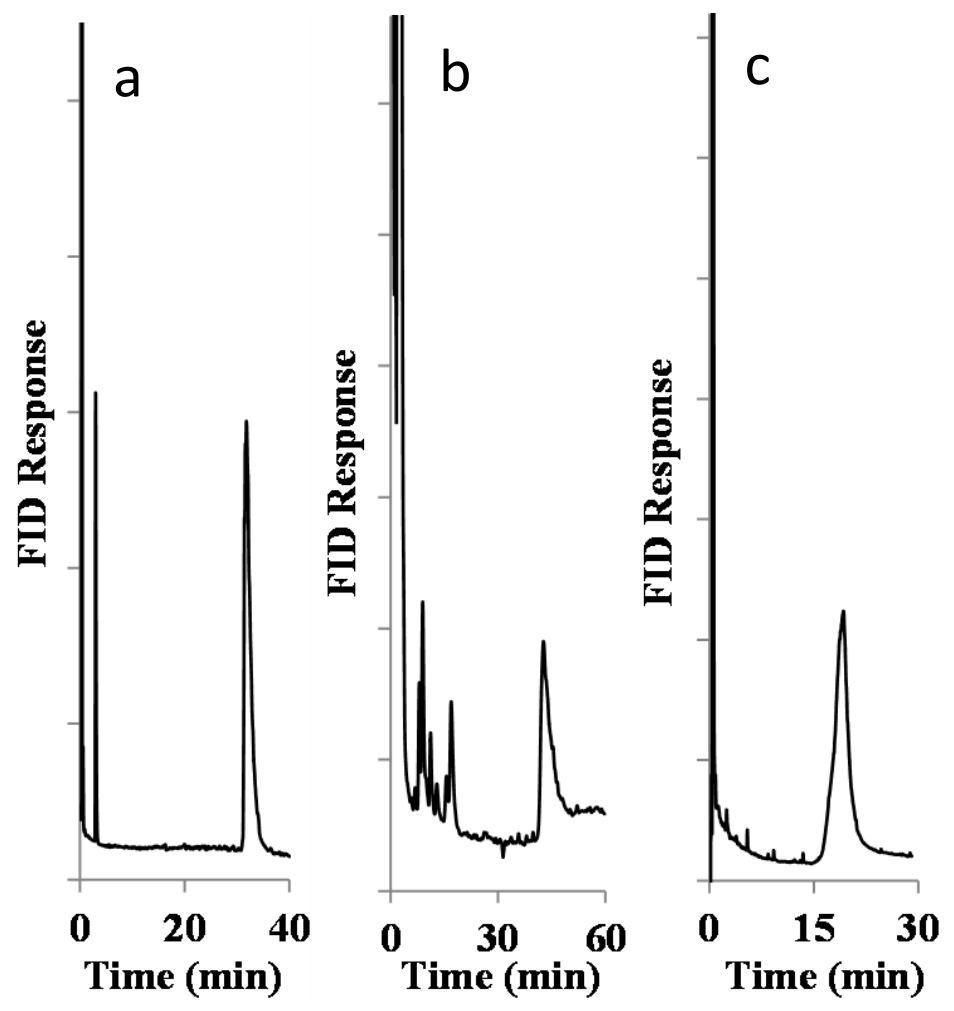

Fig. 7 

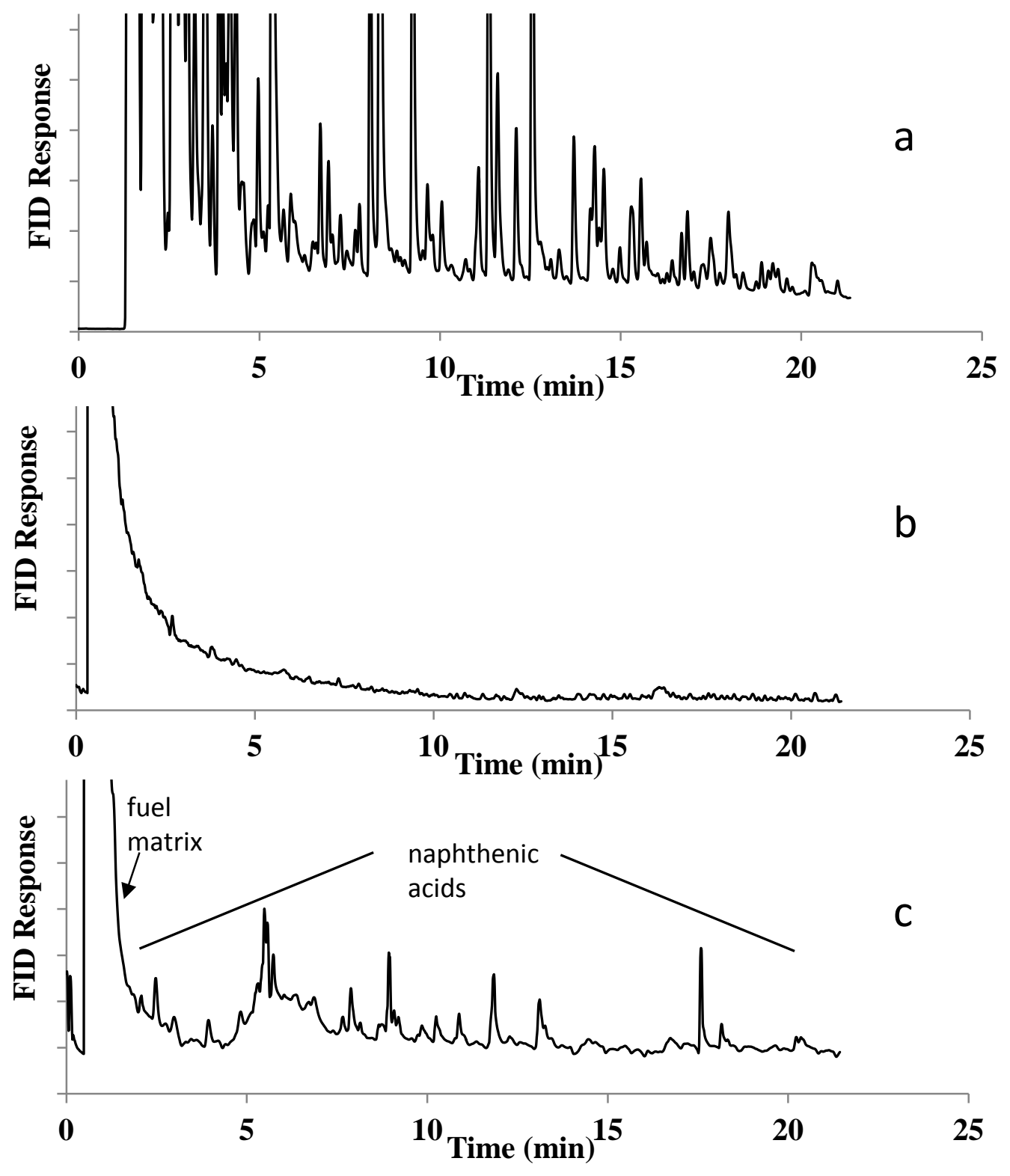

Fig. 8 